\title{
Nachricht von der in Nr. 6. des vorigen Hefts dieses Journals gedachten Sammlung von Briefen an und von L. Euler.
}

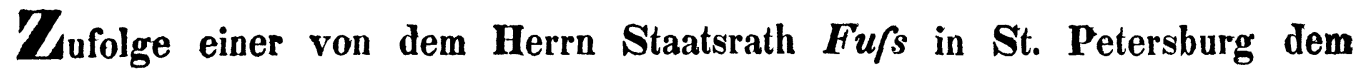
Herausgeber dieses Journals kürzlich gefälligst mitgetheilten Nachricht ist der Druck dieser Sammlung bereits ziemlich vorgeschritten. Sie wird zugleich ein systematisches Verzeichnifs der Werke Eulers enthalten. Titel und Inhalt (schreibt Herr etc. Fu/s) werden folgende sein.

"Correspondance mathématique et physique de quelques ceilebres géometres du 18 sciècle, tirée des archives centrales de Moscou et de celle de l'Académie impériale des sciences de St. Petersbourg, et précédée d'une liste complète systematique des travaux d'Euler et d'une notice sur ses écrits inédits.

I. Band. Vorrede: Ueber die Entstehung der Sammlung. Biographische und literar - historische Notizen. Nachricht über Euler's Schriften. Systematisches Verzeichnifs derselben.

Briefwechsel zwischen Euler und Goldbach, 1729-1766 (96 Briefe von Euler, 84 von Goldbach).

II. Band. Briefe Johann Bernoulli des Aeltern an Euler, 1728-1746 (14 Briefe).

Briefwechsel zwischen Nic. Bernoulli (Joh. fil.) und Goldbach, 1721 - 1725 (27 Briefe).

Briefwechsel zwischen Dan. Bernoulli und Goldbach, 1723 bis 1730 (71 Briefe).

Briefe Dan. Bernoull's an Euler, 1727-1755 (58 Briefe). Briefe desselben an Nic. Fu/s, 1773-1778 (5 Briefe).

Briefe Nic. Bernoalli's, des Neffen von Joh. und Jacob, an Euler, 1742, 1743 (4 Briefe). 
288 Nachricht von der Sammlung von Briefen an und von L. Euler.

$$
\begin{aligned}
& \text { Briefe an Euler von Naude, } 1740 \text { (1 Brief). } \\
& - \text { - - - - Clairaut, } 1740 \text { (2 Briefe). } \\
& - \text { - - - - - Cramer, 1743-1750 (7 Briefe). } \\
& - \text { - - - - Lambert, } 1758-1762 \text { (7 Briefe). }
\end{aligned}
$$

Dem 1sten Bande hoffe ich das Bild Lulers, dem 2ten das Daniel Bernoulli's beizugeben, ersteres nach dem (nach meines Vaters Ausspruch sehr ähnlichen ) Darbes'schen, von Küttner gestochenen Bilde, letzteres nach einem bei der Akademie befindlichen Oelgemälde; beide werde ich in Kupfer stechen lassen." 